\title{
tic\&société
}

Vol. 3, $n^{\circ}$ 1-2| 2009

TIC et diasporas

\section{Du brain drain à l'e-diaspora : vers une nouvelle culture du lien à l'ère du numérique}

\section{Mihaela Nedelcu}

\section{(2) OpenEdition \\ Journals}

Édition électronique

URL : http://journals.openedition.org/ticetsociete/675

DOI : 10.4000/ticetsociete.675

Éditeur

Association ARTIC

Référence électronique

Mihaela Nedelcu, «Du brain drain à l'e-diaspora : vers une nouvelle culture du lien à l'ère du

numérique », tic\&société [En ligne], Vol. 3, n 1-2 | 2009, mis en ligne le 14 décembre 2009, consulté le

19 avril 2019. URL : http://journals.openedition.org/ticetsociete/675; DOI : 10.4000/ticetsociete.675 


\title{
Du brain drain à l'e-diaspora : vers une nouvelle culture du lien à l'ère du numérique
}

\author{
Mihaela Nedelcu \\ Docteure en sociologie \\ Chargée d'enseignement et de recherche \\ Institut de Sociologie et \\ Forum Suisse pour l'étude des migrations et de la population \\ Université de Neuchâtel \\ Faubourg de l'Hôpital 27 \\ 2000 Neuchâtel, Suisse \\ mihaela.nedelcu@unine.ch
}

Docteure en sociologie, Mihaela Nedelcu est chargée d'enseignement et de recherche à l'Institut de sociologie de l'Université de Neuchâtel en Suisse et postdoctorante au Forum suisse pour l'étude des migrations et de la population. Ses principaux travaux de recherche portent sur le transnationalisme migrant, la globalisation et les processus de cosmopolitisation et en particulier, la mobilité internationale des élites et l'impact des TIC sur les processus migratoires. Elle est l'auteure du livre Le migrant online. Nouveaux modèles migratoires à l'ère du numérique, Paris, L'Harmattan, collection «Questions sociologiques », 2009. En 2004, elle a publié sous sa direction l'ouvrage collectif La mobilité internationale des compétences. Situations récentes, approches nouvelles. Actuellement, Mihaela Nedelcu codirige à l'Université de Neuchâtel (avec le professeur François Hainard) le projet de recherche Transnationalisme et TIC: nouveaux défis pour l'intégration des migrants en Suisse? L'exemple des migrations roumaines, financé par le Fonds national suisse pour la recherche scientifique (2009-2012). 


\title{
Du brain drain à l'e-diaspora : vers une nouvelle culture du lien à l'ère du numérique
}

\begin{abstract}
Résumé
Les technologies mobiles d'information et de communication produisent une transformation radicale de la façon d'être ensemble des migrants et des nonmigrants et de leur capacité d'interagir, de s'organiser transnationalement, et d'agir localement à distance. Utilisées par des migrants hautement qualifiés, dotés d'un capital humain, social et culturel élevé, elles génèrent des stratégies collaboratives novatrices et contribuent à l'émergence de nouveaux modèles d'action transnationale.

Cet article, basé sur une étude netnographique du site web www.ad-astra.ro, montre comment la diaspora scientifique roumaine se soude dans l'espace virtuel et se mobilise autour d'un objectif commun : contribuer efficacement aux processus de réforme du système éducationnel et de la recherche. Ainsi, le fonctionnement déterritorialisé articulé à l'ancrage local du réseau virtuel ont permis de transformer $A d-A s t r a$ en acteur légitime de la société civile, reconnu par les institutions de l'État roumain.
\end{abstract}

Mots-clés : Migration, e-diaspora scientifique, e-pratiques transnationales, réseaux hybrides, Roumanie, brain drain.

\begin{abstract}
Mobile information and communication technologies (MICT) are profoundly changing the way of being together of migrant and not-migrant populations, as well as their capacity to interact, to organize themselves transnationally and to act remotely at the local level. When used by highly skilled migrants, endowed with high levels of human, social and cultural capital, MICT generate innovative collaborative strategies and new patterns of transnational action.

Based on a netnographic study of the website www.ad-astra.ro, this article shows how the Romanian scientific diaspora connects in virtual space and mobilizes itself around a common aim: to efficiently improve the reform process of the education and research system. Thus, deterritorialized practices articulate with local representation in the virtual network to propel
\end{abstract}


Ad-Astra as a legitimate actor of civil society, recognized as partner by the institutions of the Romanian State.

Keywords : Migration, scientific e-diaspora, transnational e-practices, hybrid networks, Romania, brain drain.

\section{Resumen}

Las tecnologías móviles de información y de comunicación (TMIC) transforman radicalmente la forma en que se juntan las comunidades de emigrantes con las de no emigrantes. También transforman su capacidad de interaccionar, de organizarse transnacionalmente y de actuar a nivel local desde sitios remotos. Las TMIC, utilizadas por nómadas altamente cualificados, dotados de un capital humano, social y cultural elevado, generan estrategias colaborativas innovadoras a la vez que contribuyen al nacimiento de nuevos modelos de acción transnacional.

Este artículo, basado en un estudio netnográfico del sitio web www.adastra.ro, muestra la forma en que la diáspora científica rumana se junta en el espacio virtual y cómo mediante la movilización en torno a un objetivo común contribuye eficientemente a los procesos de reforma de los sistemas educativo y de investigación. Así, el funcionamiento desterritorializado articulado a las raíces locales de la red virtual ha permitido transformar adastra en un actor legítimo de la sociedad civil, reconocido por las instituciones del Estado rumano.

Palabras clave : Migración, diáspora científica, prácticas transnacionales virtuales, redes hibridas, Rumania, fuga de cerebros. 
Du brain drain à l'e-diaspora

\section{Introduction}

Le $X X X^{e}$ siècle a débuté sous le signe d'une accélération de la mondialisation, ainsi que de l'amplification et la complexification des mobilités migratoires. Par ailleurs, celles-ci se déploient aujourd'hui dans un monde marqué par la révolution numérique et l'entrée dans une ère où les technologies de l'information et de la communication (TIC) font partie intrinsèque des modes de vie quotidiens. Ces technologies contribuent à un métissage permanent des visions du monde, à l'émergence de nouvelles formes de reproduction sociale et de nouveaux modèles identitaires. Elles facilitent la mobilisation transnationale autour d'enjeux et d'objectifs locaux et spécifiques, mais aussi l'organisation d'actions locales sur la base de principes et de valeurs plus généraux. Entre les mains des migrants hautement qualifiés, dotés d'un capital humain et culturel élevé, les TIC donnent lieu à des usages et des pratiques transnationales novateurs (Nedelcu, 2004, 2008).

Cet article se penche sur la migration des scientifiques roumains en intégrant cette nouvelle réalité. Tout d'abord, un bref panorama des migrations postcommunistes des élites roumaines permet d'en saisir la diversité et l'ampleur. Ensuite, un rapide tour d'horizon théorique souligne le renouveau des perspectives avec lesquelles on aborde les migrations des cerveaux. Enfin, l'étude netnographique d'un réseau virtuel de scientifiques roumains ${ }^{1}$ - et en particulier l'observation du site web www.ad-astra.ro, de la liste de discussion qui y est associée, ainsi que l'analyse de contenu des messages échangés dans cette liste entre août 2001 et décembre $2006^{2}$ - permet d'analyser les

\footnotetext{
${ }^{1}$ L'étude des dynamiques d'un réseau virtuel oblige le sociologue à se tourner vers un terrain inédit, à approcher par écran interposé. L'observation des groupes virtuels de type liste ou forum de discussion est une nouvelle forme de "champ " anthropologique (Clifford, 1997). La netnographie (Nedelcu, 2008) permet d'investiguer ce champ d'observation volatile, qui change à coup de clic, dans lequel les configurations d'acteurs sont fluctuantes. Le chercheur peut pratiquer une observation participante des dynamiques online, lui-même engagé dans les échanges, ou simple observateur non-intrusif de ceux-ci. II peut choisir d'être acteur, " jouant » la provocation et lançant le débat vers des thèmes qui intéressent la recherche ; ou rester simple témoin de ces dynamiques, se conformant au « moule » du groupe de discussions (Héas et Poutrain, 2003). C'est cette dernière posture que nous avons préféré adopter dans l'étude du réseau virtuel de scientifiques roumains $A d-$ Astra.

${ }^{2}$ Une archive de 6432 messages a constitué la base de notre travail. Dans un premier temps, nous avons procédé à une analyse thématique des contenus de ces messages. Par ailleurs, il est intéressant de noter que certains thèmes (que nous développerons par la suite dans notre étude) sont davantage débattus que d'autres, pouvant générer des centaines de réactions et messages. Ensuite, en suivant les fréquences et les contenus des interventions des participants aux débats virtuels, nous en avons identifié les acteurs-clé. De cette façon, nous avons pu constater que seule
} 
ressorts des actions transnationales enclenchées à travers internet. Nous mettrons plus particulièrement en évidence le rôle des e-pratiques dans l'émergence d'un espace transnational multipolaire de coprésence dans lequel migrants et non-migrants se rencontrent et agissent ensemble (souvent à distance) dans une nouvelle sphère publique transnationale. Nous allons montrer ainsi que, à l'aide des TIC, la diaspora scientifique roumaine est capable de se mobiliser autour d'objectifs communs et d'influencer les processus de réforme du système éducationnel et de la recherche, en devenant un partenaire légitime de discussion pour le gouvernement roumain. Les scientifiques roumains expatriés peuvent ainsi œuvrer pour le développement du pays d'origine sans y retourner.

\section{Les migrations des élites roumaines après 1989}

L'ouverture des frontières des pays de l'Est après 1989 a conduit à une diversification des flux migratoires en provenance de Roumanie, caractérisée à la fois par une intensification des circulations migratoires à l'intérieur de l'Europe et par le développement de migrations permanentes vers le Canada, l'Australie et les États-Unis. Les dynamiques migratoires nouvellement instaurées par les migrants roumains ont prouvé que ceux-ci, malgré des décennies d'isolement politique et un imaginaire migratoire a priori peu connecté à la réalité, sont capables de s'adapter rapidement à la mobilité, de tracer des parcours originaux, de construire des espaces transnationaux et de (ré)inventer une culture du lien (Potot, 2003 ; Lazaroiu et al., 2004 ; Nedelcu, 2008).

Les mobilités des élites ont été orientées à la fois par les dynamiques mondialisées du marché et par les restrictions à la circulation des personnes imposées par les États européens. De ce fait, elles ont connu des formes diversifiées, au carrefour de plusieurs typologies: migration de cerveaux, circulation de compétences, émigration durable. Les travailleurs hautement qualifiés, et en particulier les spécialistes du secteur des technologies de l'information, ont été très nombreux à émigrer de façon définitive, peu de temps après la fin de leurs études, dans des pays comme le Canada ${ }^{3}$ et les États-Unis.

une minorité des membres du réseau - formée principalement des scientifiques roumains à l'étranger - agissent de manière systématique et engagée dans cette agora électronique. Néanmoins, les effets des actions ainsi enclenchées débordent les frontières du groupe virtuel.

${ }^{3}$ Le Canada est devenu une destination très attrayante pour les ingénieurs et les informaticiens roumains. La Roumanie se trouve depuis 1997 dans le "Top Ten » des pays-source pour l'immigration au Canada, qui a accueilli entre deux et cinq mille Roumains par année depuis 1990 (selon les rapports CIC 2001-2006). En 2002, l'année de l'apogée de la migration roumaine au Canada, la Roumanie se situait en septième position des pays-source (et premier pays-source européen), après les grands fournisseurs asiatiques d'immigrés : la Chine, l'Inde, le Pakistan, les Philippines, l'Iran et la Corée du Sud. Les données partielles du recensement canadien de 2002 révèlent la présence de 60520 Roumains au Canada. 
La proportion des diplômés d'université en migration a augmenté de $6 \%$ en 1990 à $23 \%$ en 2000. En comparaison, ceci représente le double du pourcentage (d'environ 10\%) des diplômés d'universités dans la population adulte totale (Lazaroiu et al., 2004). Certaines études récentes indiquent que la proportion des diplômés d'universités a représenté même une part beaucoup plus importante des migrations internationales des Roumains, en dépassant les $50 \%$ (Salt et al., 2005; OCDE, 2001). Les migrations des scientifiques ont également pris de l'ampleur, dans le contexte d'un environnement de recherche peu compétitif, des infrastructures insuffisantes et des politiques de recherche peu favorables à la performance (Haiduc, 2001 ; Frangopol, 2002). Si, en 1989, la Roumanie disposait d'environ 150000 personnes actives dans le domaine de la recherche ${ }^{4}$, ces effectifs ont diminué jusqu'à 19726 en 2001, pour réaugmenter à 38433 en 2002, et environ 30000 en 2004. En 2001, la Roumanie se situait en avant-dernière position en Europe, avec seulement $0,39 \%$ de la force de travail constituée de chercheurs tandis que la moyenne européenne était de $1,39 \%$. Par extrapolation des résultats de certaines études récentes, il est à croire qu'environ $64 \%$ des scientifiques roumains, reconnus comme ayant des publications dans des revues cotées, résident à l'étranger (Florian, 2004). Ceci dans les conditions où, en 2004, la Roumanie a consacré 235 millions d'euros à ses dépenses de recherche et de développement, soit seulement $0,45 \%$ de son PIB - 0,29\% financés par l'État et $0,13 \%$ par les entreprises (selon les données OCDE 2005).

Cette réalité reste inquiétante pour un pays qui a besoin de toutes ses ressources hautement qualifiées pour répondre aux défis actuels de l'intégration européenne, d'autant plus que la Roumanie a connu pendant les cinq dernières années une croissance économique sans précédent. En même temps, suite à l'adhésion de dix pays ex-communistes à l'Union européenne, la donne européenne est en train de changer. Les scientifiques de ces pays, y inclus la Roumanie, font désormais partie de I'ERA (European Research Area). Leur insertion dans des réseaux scientifiques d'excellence constitue, de ce fait, un facteur clé pour la production d'un champ transnational de mobilité, qui se nourrit de la circulation des informations, des idées, des productions scientifiques, des technologies, des expertises et des personnes.

\footnotetext{
${ }^{4}$ Selon la législation roumaine (loi 319/2003) et la nomenclature roumaine des fonctions et métiers, les chercheurs font partie de la catégorie " personnel de recherche-développement » qui inclut non seulement les "scientifiques", mais également les "cadres didactiques universitaires " et le « personnel technique auxiliaire ».
} 


\section{Du « brain drain » à l'« option diaspora » ou comment les migrations des cerveaux peuvent- elles bénéficier au pays d'origine?}

Tant les acteurs politiques que les chercheurs en sciences sociales s'accordent aujourd'hui à reconnaître que les migrations des cerveaux ne représentent pas toujours et irrémédiablement une perte pour les pays d'origine (Salt et Findlay, 1989; Gaillard et Gaillard, 2002 ; Lowell et Findlay, 2002). II existe désormais un intérêt croissant non seulement pour les conséquences néfastes de ces migrations, mais aussi pour leurs " externalités " positives et leur contribution directe au développement des pays d'origine. Ces derniers s'adaptent et adoptent des mesures politiques leur permettant d'en tirer le meilleur profit (Wickramasekara, 2004 ; de Haas, 2006 ; Barré et al., 2003).

Le paradigme du "brain drain " a été ainsi remplacé par une nouvelle grammaire des migrations hautement qualifiées (Meyer et Charum, 1995). Dans l'actuel contexte économique, politique et technologique, de nouveaux concepts ou de nouvelles expressions décrivent mieux la dynamique multipolaire, accélérée et diversifiée de ces flux. Des termes comme professional transients, brain gain, reverse transfer of technology, transit brain drain, delayed return, skilled transients, brain mobility, brain exchange, diaspora network (Gaillard et Gaillard, 1998 ; Findlay, 1988 ; Saxenian, 2000 ; Barré et al., 2003) signalent l'apparition de nouvelles approches centrées sur les échanges qui se développent au sein de réseaux multiples, scientifiques et technologiques, entre les différents pôles de la migration. Ces échanges, amplifiés et accélérés par les TIC, ont conduit à l'émergence de réseaux diasporiques qui véhiculent des ressources directement exploitables par les pays d'origine. La capitalisation de ces ressources peut prendre des formes variées : transferts de fonds, création de réseaux d'affaires et de commerce entre les lieux d'accueil et ceux d'origine, transfert d'expertise et de technologies à travers des réseaux scientifiques (Meyer et Hernandez, 2004; Barré et al., 2003; Gaillard et Gaillard, 2002; Lowell et Findlay, 2002).

Une nouvelle vision de la diaspora voit le jour lorsque celle-ci prend forme autour de l'action et de la projection dans un avenir pour lequel les élites scientifiques expatriées s'investissent (Meyer et Hernandez, 2004). En outre, l'activisme des diasporas techniques et scientifiques se traduit dans de nouveaux modèles collectifs d'action qui se propagent à travers les e-réseaux des migrants qualifiés. En étudiant les différentes configurations et activités de ces réseaux, Jean-Baptiste Meyer et Valeria Hernandez (2004) ont montré qu'elles engagent des moyens divers (matériels, symboliques et politiques) et se 
déploient dans des structures (réseau professionnel, association, amicale, fédération...) qui ne jouissent ni du même poids, ni de la même reconnaissance dans les rapports avec les institutions des pays d'origine. Les logiques de réinvestissement par rapport au pays d'origine qui s'en suivent varient aussi : bénévolat, philanthropie, volontariat, mais aussi investissement économique et engagement civique (Barré et al., 2003). En outre, plusieurs conditions doivent être réunies pour garantir une continuité des activités des diasporas techniques et scientifiques dans le temps et assurer la durabilité de leur impact sur le développement du pays d'origine: l'ancrage local entraînant un dynamisme associatif efficace, une forte capacité de mobilisation, ainsi qu'une visibilité maximale; la fédération des différents pôles diasporiques afin d'assurer une capitalisation des ressources expatriées; la création de canaux efficaces de communication, notamment à travers les technologies mobiles (internet, téléphonie mobile...) qui permettent d'agir à distance en temps réel (Meyer et Hernandez, 2004). En même temps, les États d'origine multiplient aussi leurs efforts. En créant des espaces de rencontre et en encourageant la participation et l'expression d'une citoyenneté déterritorialisée et flexible, ils s'affirment en tant qu'acteurs transnationaux et misent sur une politique de " nation globale » qui englobe les nationaux expatriés. La réussite de cette politique reste cependant conditionnée par l'équilibre indispensable entre les stratégies individuelles et étatiques, entre l'intérêt privé et public.

L'étude de cas décrite par la suite met en évidence comment un réseau virtuel de jeunes chercheurs roumains a permis la maturation d'une e-diaspora scientifique et s'est imposé en tant qu'acteur légitime dans les jeux politiques autour de la réforme de l'enseignement et de la recherche en Roumanie.

\section{3. www.ad-astra.ro : le creuset électronique d'un projet d'action transnationale des scientifiques roumains}

Le projet Ad-Astra a germé lors de la rencontre de plusieurs doctorants et scientifiques dans le cadre du premier Forum des jeunes Roumains éduqués à l'étranger. Ce forum, organisé en 2000 par le Président de la Roumanie et l'ambassade des États-Unis à Bucarest, avait comme but d'inciter ces jeunes qualifiés au retour ou, du moins, de les sensibiliser à un effort de réinvestissement pour la Roumanie. L'initiative, qu'on pourrait qualifier de transnationale «par le haut », resta toutefois éphémère et ponctuelle, car elle n'a pas été suivie dans l'immédiat d'une politique soutenue, cohérente et articulée à l'égard des migrants hautement qualifiés. 


\section{1. "Per aspera ad astra » : la création d'un réseau virtuel de jeunes chercheurs roumains}

Ce lieu de rencontre a permis toutefois la création de liens informels entre les jeunes scientifiques participant au forum ainsi que l'identification d'intérêts communs de recherche et d'action. Par ailleurs, quelques participants ont adhéré à l'initiative d'un confrère, chercheur dans un institut américain d'océanographie, visant à créer un journal en ligne, première "agora » délocalisée des scientifiques roumains engagés dans la production scientifique mondiale. Après une année de vifs échanges par courrier électronique, pendant laquelle les initiateurs (dispersés aux États-Unis, en Suède, en France, en Roumanie...) ont débattu de la meilleure forme à donner à ce projet, le site web www.ad-astra.ro a vu le jour. Créé au départ comme support du journal scientifique $A d-A s t r a$, il est rapidement devenu une plate-forme d'échanges scientifiques et de mise en commun d'expertise et de compétences. Le site web héberge entre autres une base de données Who's who dans la science roumaine (structurée par domaine scientifique), une bibliothèque de la science roumaine Science Library, un agenda de nouveautés, un calendrier à jour des événements scientifiques et une bourse aux financements de recherche.

Si le roumain est la langue utilisée en règle générale dans les échanges sur la liste de discussions associée à ce site web, la principale interface de ce dernier est en anglais. En outre, les premiers numéros du journal en ligne ont été édités uniquement en anglais et, seulement depuis récemment, on accepte aussi des articles en roumain. Ces choix s'inscrivent dans une stratégie générale qui vise une audience et un impact à la fois nationaux et internationaux. 
Du brain drain à l'e-diaspora

\section{Figure 1}

Structure et fonctionnalités du site web Ad-Astra (état au 5 novembre 2007)

\begin{tabular}{|c|c|}
\hline 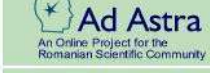 & \\
\hline $\begin{array}{l}\text { Ad Astra Journal } \\
\text { Science librany } \\
\text { White book } \\
\text { University rankings } \\
\text { Who's who } \\
\frac{\text { Publications }}{\text { Theses and dissertations }} \\
\text { Ad Astra association } \\
\text { Links } \\
\text { News } \\
\text { Events } \\
\text { Funding opportunities } \\
\text { Login } \\
\text { Reqistration } \\
\text { P Română }\end{array}$ & $\begin{array}{l}\text { About us } \\
\text { Ad Astra is an online project devoted to the Romanian scientific community that is } \\
\text { programmatically aimed at the young generation. } \\
\text { Our goals are: } \\
\text { - to showc ase modern science, performed either in Romania or by Romanian scientists } \\
\text { abroad; } \\
\text { - to present latest scientific paradigms and methodological concepts to be used as } \\
\text { benchmarks for objectively estimating the state of science and education in Roranaia; } \\
\text { - to initiate and maintain a fux of information that will facilitate scientific cooperation within } \\
\text { the Romanian scientific communities, } \\
\text { to encourage and advise young researchers in Romania; } \\
\text { to provide an open discussion forum on science and education policies, with the declared } \\
\text { aim of presenting coherent reform proposals to the Romanian political establishment. } \\
\text { Participation to the project is encouraged for anybody with interests in science! } \\
\text { The Ad Astra website was launched in Frebruary } 2002 \text { as a volunteer project, by Liviu Giosan and } \\
\text { Razvan Florian. Ad Astra incorporated in July } 24,2002 \text { as a non-profit association, and now } \\
\text { administers the project. } \\
\text { Ad Astra Association } \\
\text { Ad Astra events and activities } \\
\text { Ad Astra in press }\end{array}$ \\
\hline$\frac{\text { About us }}{\text { (ad Ad Astra }}=\frac{\text { Contributel }}{2001-2006} \cdot$ Spor & $\frac{\text { isclaimer }}{\text { Anxia }}$ Contact \\
\hline
\end{tabular}

L'accès aux informations diffusées à travers le site web est ouvert à tous. Par ailleurs, tout étudiant, jeune chercheur ou chercheur confirmé peut adhérer librement et devenir membre de cette communauté online en enregistrant ses principales publications scientifiques. Cependant, seules les personnes ayant publié dans des revues d'impact international peuvent figurer dans le Who's who.

Parmi les 143 membres qui s'y sont inscrits après une année de fonctionnement, 48 résidaient aux États-Unis, 46 en Roumanie, 12 en France, 8 en Allemagne, 5 au Canada et en Grande Bretagne, 3 en Grèce, Pays Bas et Suisse, 2 en Italie, Australie et Moldavie, 1 en Suède, Belgique, Irlande et Autriche $^{5}$. Sans refléter la dispersion des scientifiques roumains dans le monde, cette répartition mettait néanmoins en évidence l'attrait qu'exercent les États-

\footnotetext{
${ }^{5}$ Selon l'interrogation de cette base de données le 27 novembre 2002. http://www.adastra.ro/whoswho/?lang=ro
} 
Unis. L'impact ultérieur de ce projet et la large reconnaissance d'Ad-Astra dans les milieux scientifiques et académiques roumains se sont traduits dans une nouvelle version 2006 plus étoffée du Who's who, regroupant plus de 650 scientifiques roumains reconnus internationalement et faisant état de plus de 3700 de leurs publications ${ }^{6}$. En outre, plus de la moitié de ses membres résident en Roumanie (graphique 1). Cette base de données constitue actuellement un réservoir réel de compétences et peut servir à l'initiation de collaborations entre les chercheurs roumains.

Graphique 1 : Répartition des membres d'Ad-Astra par pays de résidence (état au 5 novembre 2007)

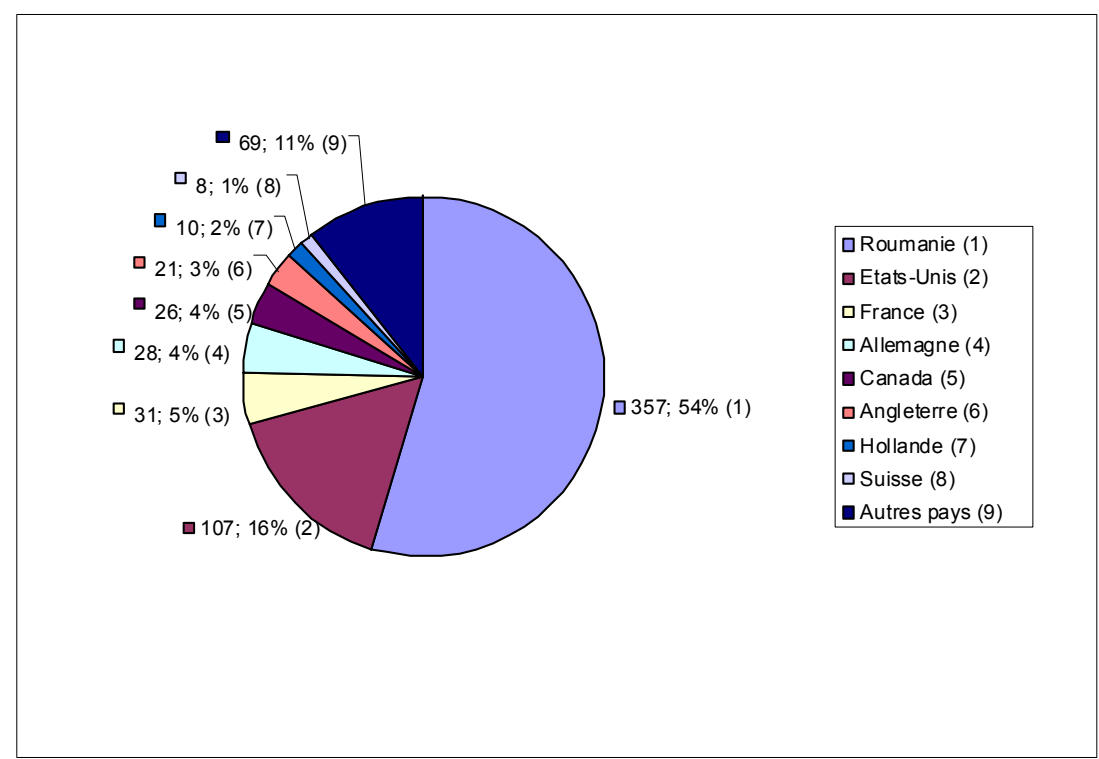

Des 14 éditeurs en ligne ${ }^{7}, 2$ résident en Roumanie, 5 aux États-Unis, 3 en France, 1 en Suède, 1 en Suisse, 1 en Australie, 1 en Grande-Bretagne (état au 16 novembre 2006). Tous sont de jeunes chercheurs actifs dans des instituts de recherche occidentaux prestigieux, membres des réseaux scientifiques de haut

\footnotetext{
${ }^{6}$ En réalité, Ad Astra dispose d'une base de données d'environ 1400 chercheurs roumains, inscrits indifféremment de leur performance scientifique.

${ }^{7}$ Spécialistes dans des disciplines aussi diverses que les mathématiques, la chimie, la physique, l'informatique, la biologie ou les sciences humaines et sociales, ces personnes mobilisent leurs réseaux et incitent des collègues à écrire dans le journal Ad-Astra. Ils fonctionnent également comme experts, garants de la publication de travaux de qualité.
} 
niveau, bien intégrés socialement et professionnellement dans leurs pays d'accueil respectifs.

II convient aussi de noter certaines polarités disciplinaires, les domaines scientifiques et techniques étant de loin surreprésentés. La physique, la chimie, les sciences de l'ingénierie, la biologie dominent les disciplines d'appartenance des membres du Who's who, tandis que les sciences humaines, politiques et sociales sont très faiblement représentées (graphique 2).

Graphique 2 : Répartition des membres d'Ad-Astra par domaine scientifique (état au 5 novembre 2007)

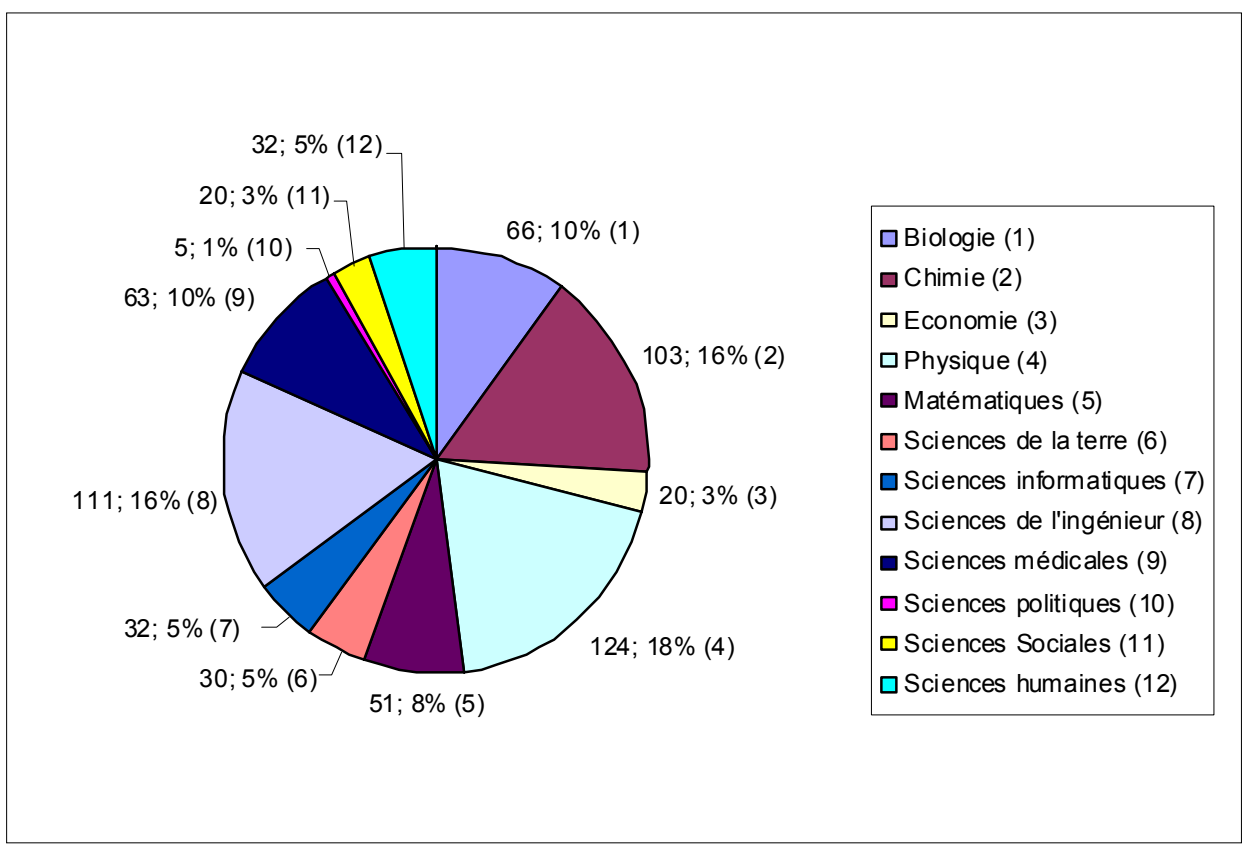

\subsection{Les thèmes-clés structurant le débat transnational}

Le site web a constitué un outil central pour la diffusion des idées du groupe d'initiative et la mise en réseau d'un grand nombre de scientifiques d'origine roumaine. Cependant, seulement un groupe restreint de membres a participé constamment aux débats médiatisés par l'e-mail. Très actif, il a soudé le réseau autour d'un objectif commun: contribuer à la restructuration du système 
roumain de la recherche. II a aussi alimenté assidûment le contenu du journal $A d$-Astra, tant en idées qu'en études scientifiques ${ }^{8}$. Plusieurs thèmes ont été soumis au débat au sein de ce groupe de discussions. À titre d'exemple, une des premières initiatives a consisté dans la réalisation d'une radiographie qui se voulait aussi exhaustive que possible du système roumain d'admission au doctorat, complétée par une étude sur le financement de la recherche et les critères d'occupation des postes de professeurs d'université. Les quelques 500 messages qui ont circulé entre les mois de mars et de mai 2003 dans la liste de discussions ont abouti à la rédaction d'un document intitulé « L'état de la recherche I. Le doctorat ». Celui-ci représente une synthèse des exigences de la formation doctorale en Roumanie, s'articulant autour des impératifs d'excellence du marché mondial de la recherche et du travail. Ce texte traduit une prise de position critique de ses auteurs. Ceux-ci jugeaient d'une manière sévère le système de doctorat existant de même que le projet de la nouvelle loi de l'enseignement qui conservait une formation doctorale tributaire d'un héritage archaïque favorable à l'« inertie » scientifique. Ce premier rapport d'Ad-Astra a constitué un signal d'alarme d'autant plus inquiétant qu'il venait de l'intérieur de la communauté scientifique. Publié dans le premier quotidien national et le journal Ad-Astra online, repris par d'autres groupes de discussion, ce texte critiquait sans réserve les initiatives gouvernementales jugées souvent superficielles.

À la suite de ces premières réflexions qui ont tracé la voie d'action de ce réseau de jeunes scientifiques roumains, d'autres thèmes ont émergé et en ont orienté l'activité ultérieure: l'analyse de la construction du budget de la recherche et de son utilisation, la critique des critères d'évaluation de la performance des universités, la proposition d'un nouveau règlement d'admission au doctorat, la participation à des conférences au sujet de la migration de jeunes chercheurs, la mise sur pied d'un programme de vulgarisation de la science par des conférences données par des membres de l'association dans des collèges et des lycées. Ce réseau virtuel a produit de la sorte une série de documents: des synthèses des difficultés, voire des failles des processus de

\footnotetext{
${ }^{8}$ Mentionnons quelques-unes des premières publications auxquelles ont donné lieu les études lancées par les membres du groupe: Giosan L. et T.I. Oprea, 2002, "Stiinta in Romania postcomunista: viitorul nu este luminos ", Ad Astra 1 (2). http://www.adastra.ro/journal/editorial ro.pdf; Oprea T.I., 2001, « De veghe in cercetarea romaneasca : Eseuri de filozofia cercetarii », Timisoara, Ed. Mirton. http://www.adastra.ro/library/books/oprea de veghe.pdf; Florian R., 2004, "Migratia cercetatorilor romani. Situatia actuala, cauze, solutii », Ad Astra 3 (2). http://www.ad-astra.ro/journal/6/florian migratia.pdf ; Buhai S., 2004, "Conditia cercetatorului. Intre realitatea de acasa si idealul din afara ", Ad Astra 3 (2). http://www.ad-astra.ro/journal/6/buhai conditia cercetatorului.pdf; Szedlacsek S., « Zece cai de a atrage cercetatorii romani spre Romania ", Ad Astra 3 (2) 2004. http://www.adastra.ro/journal/6/szedlacsek.pdf. Pour une liste complète des articles le lecteur peut consulter les numéros du journal en ligne http://www.ad-astra.ro/journal.
} 
mise au concours et des programmes de financement de la recherche, la proposition d'un «index Ad-Astra » permettant l'introduction des critères bibliométriques d'évaluation de la capacité de recherche des universités et des chercheurs, la réalisation d'un classement des universités roumaines comparable au classement Shanghai, une synthèse de bonnes pratiques internationales dans le domaine du financement compétitif de la recherche ${ }^{9}$.

À travers ses études, Ad-Astra a réalisé un état des lieux précis de la condition de chercheur en Roumanie, visant un double objectif : d'une part, la dénonciation des résistances auxquelles les chercheurs compétents sont confrontés, notamment le manque de transparence, la corruption, les critères aléatoires ou le manque de professionnalisme des évaluateurs; d'autre part, la proposition d'alternatives aux politiques du gouvernement, dans le but de transformer en profondeur le système de la recherche. En outre, à partir d'une analyse des politiques développées dans d'autres pays ${ }^{10}$ et de leur propre expérience en matière de formation à la recherche dans les grands instituts universitaires occidentaux, les participants aux échanges résidant à l'étranger ont continuellement partagé une expertise scientifique et sociale qu'ils souhaitent mettre au service de réformes en Roumanie.

Par ailleurs, il faut noter que les scientifiques expatriés se trouvent aussi parmi les membres les plus actifs du réseau virtuel ${ }^{11}$. Le débat passionné des difficultés rencontrées par les jeunes chercheurs en Roumanie témoigne de leur réelle préoccupation pour l'avenir du système roumain d'enseignement et de recherche, ainsi que de leur volonté à s'engager dans une action commune, susceptible d'enclencher un processus de réforme, et ceci, malgré une situation encore trop peu attractive et favorable au retour des chercheurs roumains expatriés.

\footnotetext{
${ }_{10}^{9}$ Voir http://www.ad-astra.ro/phare/studii.php.

"Le modèle hongrois " a suscité, par exemple, tout un débat sur l'importance des mesures incitatives prises par l'État pour stimuler l'intégration des chercheurs autochtones à un système de recherche compétitif, de niveau mondial.

${ }^{11} \mathrm{Si}$ le réseau $A d$-Astra a été initialement créé par un groupe restreint de scientifiques roumains expatriés, en réponse à leurs besoins de se (re)connecter aux milieux roumains de la recherche et de créer des ponts entre eux et les collègues du pays, ils ne représentent aujourd'hui, comme le montre le graphique 1 , qu'une minorité des membres et sympathisants de ce réseau. Cependant, l'impact de la participation de ces scientifiques expatriés aux activités du réseau continue d'être significatif pour la perpétuation de celui-ci. En particulier, le noyau des acteurs-clés d'Ad-Astra est constitué d'une dizaine de personnes très dynamiques et attachées aux idéaux du réseau, la plupart se trouvant à l'étranger.
} 
Mihaela NEDELCU

\subsection{L'ancrage local d'un réseau délocalisé : comment une entité électronique devient-elle un véritable acteur de la société civile roumaine?}

L'utilisation des TIC, et en particulier de l'internet et de ses facilités (site web, email, groupe de discussion), constitue la clé de voûte du fonctionnement transnational de ce réseau. Quelques dizaines de chercheurs roumains ont participé à cette dynamique déployée dans l'espace virtuel. En se mobilisant pour agir en faveur de la réforme de la recherche dans le pays d'origine, ils articulent leur vocation scientifique à un engagement civique. Support d'une expertise collective produite dans un espace social déterritorialisé, cette agora virtuelle a fonctionné comme une plateforme d'action pour des scientifiques migrants et non-migrants réunis autour d'un but commun.

Mais cela ne suffit pas pour créer une vraie prise de conscience et une reconnaissance politique des réalités signalées par ce réseau de chercheurs à travers son journal en ligne et son site web. Ad-Astra a cherché par conséquent un outil permettant d'investir l'espace public, de diffuser les résultats de ses rapports et analyses à large échelle et de produire un débat public attirant l'attention des milieux décisionnels roumains. En quête d'une réelle légitimité d'action, le réseau Ad-Astra s'est doté d'une personnalité juridique, en fondant une organisation non-gouvernementale (ONG), enregistrée en Roumanie en 2002. Parmi ses objectifs : soutenir et promouvoir la science roumaine moderne, qu'elle soit produite en Roumanie ou à l'étranger par des chercheurs d'origine roumaine; promouvoir les droits et les intérêts des chercheurs d'origine roumaine, notamment ceux de la nouvelle génération ; faciliter la communication et la collaboration entre les chercheurs roumains du pays et de l'étranger; soutenir et faciliter l'intégration de la recherche roumaine au système scientifique européen et mondial; contribuer à la réforme du système d'enseignement et de la recherche en Roumanie ${ }^{12}$.

En février 2009, l'association Ad-Astra réunissait 66 membres, tous des chercheurs confirmés et reconnus dans leurs domaines respectifs ${ }^{13}$, dont une trentaine résident et travaillent à l'étranger. Cette institutionnalisation de la mise en réseau des scientifiques roumains à travers internet représente de fait une capitalisation politique des ressources sociales, humaines et culturelles dont ils sont porteurs. L'ancrage national constitue la principale clé d'action qui rend effectif l'impact des réseaux à distance. Le réinvestissement des scientifiques

\footnotetext{
${ }^{12}$ Selon les statuts d'Ad Astra, en ligne à http://www.ad-astra.ro/asso/statut.php

13 La liste des membres de l'association peut être consultée à l'adresse: http://www.adastra.ro/asso/members.php?lang=ro
} 
dans la société d'origine se transforme ainsi d'initiative incitative en action performante.

Par ailleurs, pour réussir à se faire entendre par des acteurs décisionnels, Ad-Astra a multiplié ses interventions dans l'arène publique: publication d'analyses dans des quotidiens nationaux avec une grande audience ${ }^{14}$, interviews à la radio, participation à des ateliers et à d'autres débats au sein de la société civile. Portant sur des thématiques liées à l'intégration scientifique de la Roumanie à l'Europe, au développement du domaine des technologies de l'information, à la migration des spécialistes et à la réforme de l'enseignement supérieur et de la recherche, ces discussions ont contribué à faire connaître l'activité et la position du réseau. L'association a réalisé entre autres un "Livre blanc " de la recherche scientifique en Roumanie à partir des données Thomson $|S|^{15}$, mises à jour annuellement. II s'agit plus précisément d'une base de données accessible en ligne, qui répertorie les publications d'importance internationale, produites par des chercheurs roumains ou d'origine roumaine au cours des cinq dernières années. Cette base de données peut être consultée selon plusieurs critères (domaine, année, université, individu) et elle constitue un outil, construit selon une méthodologie transparente, permettant une évaluation objective de la situation réelle de la recherche roumaine. L'accès à cette base de données est public et gratuit à partir du website d'Ad-Astra. Encore perfectible, cet outil a révélé qu'environ 9000 chercheurs roumains, tous domaines confondus, soit $34 \%$ du nombre total de chercheurs, ont publié au moins un article dans une revue ISI pendant les cinq dernières années. À partir de ces données, Ad-Astra a montré aussi que plus de $53 \%$ des experts gouvernementaux du programme «Recherche d'excellence » (CEEX) chargés d'évaluer les projets de recherche et plus de $45 \%$ des directeurs de projets financés dans ce cadre n'ont publié aucun article coté ISI entre 2001 et $2005^{16}$. En outre, sur la base des résultats scientifiques ISI du corps professoral des universités, l'association réalise chaque année, depuis 2005, un classement des universités roumaines.

\footnotetext{
${ }_{15}^{14}$ II s'agit principalement de Evenimentul Zilei, Ziua, Romania Libera et Revista 22.

${ }^{15}$ Les chercheurs roumains d'Ad-Astra ont proposé des scores de performance établis sur la base des critères internationalement acceptés par la communauté scientifique internationale, notamment selon les données de l'Institute of Scientific Information (ISI), institution américaine qui répertorie environ 9000 revues et publications scientifiques considérées comme mainstream. (http://www.isinet.com, voire aussi ISI Web of Knowledge, http://www.isiknowledge.com). Dans ses analyses, le réseau Ad-Astra a montré que, selon les données ISI disponibles pour 2002, la Roumanie ne se situait qu'en $66^{\mathrm{e}}$ position dans le classement mondial de la production scientifique, avec seulement 0,77 publications scientifiques pour mille habitants, dernière dans le peloton des pays de l'Europe de l'Est. Source : http://www.ad-astra.ro/cartea-alba/?lang=ro

${ }^{16}$ Selon le rapport Ad-Astra: Florian, Razvan (2006) "Oamenii de stiinta din Romania si recunoasterea rezultatelor lor », Ad Astra 5 (1), 2006, www.ad-astra.ro/journal
} 


\subsection{L'imbrication virtuel/réel, local/transnational et l'instauration des bonnes pratiques : les clés d'une action transnationale efficace}

Si l'association Ad-Astra possède un siège social correspondant de fait à l'emplacement technique du site web (à Cluj-Napoca), les formes d'interaction et d'action au sein de cette ONG restent néanmoins dictées par la dispersion géographique de ses membres. Ceci fait d'ailleurs sa particularité. Dès le début il a été ainsi stipulé dans les statuts de l'association que l'assemblée générale peut se dérouler à l'aide d'outils électroniques de communication interactive à distance (voire internet). La liste officielle des discussions, ad astra members@yahoogroups.com est ainsi reconnue comme «lieu » principal de rencontre de ses membres. Les discussions par e-mail sont assimilées à des débats d'une session continue de l'assemblée générale. Un règlement de fonctionnement interne propose également des principes clairs de bonne conduite, le conseil directeur de l'association pouvant intervenir pour arbitrer d'éventuelles divergences ou situations conflictuelles. La procédure de prise de décisions fait l'objet d'un chapitre à part du règlement. Tout membre dispose du droit de proposer un débat sur une thématique spécifique. II peut aussi demander que celle-ci soit examinée et qu'une décision soit prise à ce propos (prise de position, publication d'un rapport, communiqué de presse...). La formulation de la décision est débattue par les membres sur la liste de discussion au moins pendant 48 heures et, en tout cas, jusqu'à ce que toutes les objections formulées sur sa clarté soient levées. La décision est ensuite soumise à une procédure de vote, par la création d'un sondage (poll). Un délai de minimum cinq jours est accordé aux membres pour s'exprimer. Aucune action impliquant l'association ne peut ainsi être engagée sans qu'une décision soit prise selon cette procédure de consultation sur internet.

L'institution de règles transparentes et unanimement reconnues par les membres de cette communauté virtuelle a garanti l'efficacité des interactions, imposant une tenue cohérente et productive des débats. Les «bonnes pratiques » instaurées dans ce groupe semblent être garantes de sa pérennité. Elles s'expliquent par plusieurs facteurs : d'abord, l'homophilie des statuts et des valeurs (Lazarfeld et Merton, 1954) des membres du réseau, ces derniers partageant des idéaux communs qui orientent leur participation dans le réseau ; puis, la transparence exigée sur les identités des participants et la restriction de l'accès à la liste de discussion aux seuls membres de l'association; ensuite, la définition commune d'un objectif collectif du regroupement virtuel; enfin l'institution d'une charte et des règles précises du fonctionnement du groupe de discussion qui a assuré un contrôle implicite de la conduite de ses membres. De la sorte, le climat de confiance instauré dans ce réseau virtuel a rendu possibles des échanges focalisés, favorisant une décision de qualité. Par ailleurs, cet 
espace social transnational tiers a pu encourager des discussions et des critiques qui autrement n'auraient pu être formulées ailleurs.

En même temps, les membres du réseau résidant en Roumanie ont un rôle important à accomplir dans la mise en application dans ce pays des initiatives " virtuelles » développées sur internet. Ils constituent l'interface réelle entre une entité transnationale, physiquement déterritorialisée, et les autorités roumaines, dont le ministère de l'Éducation et de la recherche, les universités et instituts de recherche, et des représentants de la société civile, ou des médias ${ }^{17}$. Ils ont pu ainsi asseoir la légitimité de leurs actions sur le capital d'expertise de leurs collègues de l'étranger, opposant à l'inertie locale le dynamisme et l'esprit de compétition insufflés from outside. De cette façon, Ad-Astra a permis l'émergence et la consolidation d'une identité collective du groupe de scientifiques roumains réunis dans l'espace virtuel, tout en développant une vision commune du changement qu'ils aimeraient voir se produire dans le domaine de la recherche en Roumanie.

\subsection{Légitimité et reconnaissance dans le champ de l'action politique}

Sur la base de ce capital, les scientifiques roumains exigent aujourd'hui de participer à l'élaboration de la stratégie de réforme et d'être associés aux décisions. Les prises de position auprès d'acteurs institutionnels ont été fermes, solidement construites et argumentées. L'association a interpellé constamment les autorités politiques nationales au sujet des blocages et des défaillances du processus de réforme ${ }^{18}$. L'engagement civique de ses membres, le monitoring de qualité qu'elle a initié, et l'insistance de ses interventions dans l'espace public, ont abouti, après quelques années de militantisme, à des résultats significatifs. Ad-Astra a été sollicité en 2005 par le ministère de l'Éducation et de la recherche pour proposer de nouvelles procédures d'évaluation. Le Conseil national de la recherche scientifique de l'enseignement supérieur (CNCSIS) a introduit dans ses procédures des critères supplémentaires d'évaluation de

\footnotetext{
${ }^{17}$ Un jeune informaticien résidant en Roumanie, membre fondateur du réseau et de l'association, est ainsi devenu le directeur exécutif et le porte-parole du groupe en diffusant les intérêts de celui-ci dans le pays. D'autres chercheurs roumains ont (re)présenté le point de vue de l'association dans le cadre de différents débats publics (conférences, tables-rondes...), à tour de rôle et selon leurs disponibilités.

${ }_{18}$ Ad-Astra a notamment envoyé des lettres ouvertes au premier ministre, à la commission parlementaire et au ministre de l'Éducation et de la recherche, accompagnées de synthèses et de rapports mettant en évidence des directions prioritaires d'action pour améliorer rapidement la qualité de la recherche produite en Roumanie. Elle a aussi contesté la nomination à la direction de l'Autorité nationale pour la recherche scientifique (ANCS) d'un secrétaire d'État qui ne rencontrait pas les critères minimaux de performance (un seul article ISI pendant une carrière de professeur de plus de 15 ans).
} 
l'activité de recherche, de sélection et de financement des projets, ainsi que des promotions universitaires. Suite aux demandes récurrentes formulées par AdAstra à propos de la transparence et de la publication de certaines informations d'intérêt public, le ministère de l'Éducation et de la recherche a commencé à publier depuis juillet 2007 les nominations aux postes de professeur et de maître de conférences dans les universités roumaines. Les classements des universités et les scores appréciant l'activité de recherche des scientifiques roumains proposés par $A d$-Astra sont devenus, dans une large mesure, des références qui comptent pour la communauté scientifique roumaine, qui a intégré ces indicateurs dans les pratiques d'évaluation de la recherche. En outre, l'association a été approchée par d'autres groupes de la société civile, tels le Forum académique roumain (FAR) et le Forum pour la stratégie et la réforme (FORS), préoccupés d'assainir le système de recherche roumain. Ce rapprochement a donné lieu à quelques actions coordonnées. En 2006, l'association a bénéficié également d'un financement de l'Union européenne pour son projet « Transparence et objectivité dans la gestion de la recherche en Roumanie », dans le cadre du programme PHARE sur « La consolidation de la société civile en Roumanie ».

Toutefois, si la pertinence des prises de position d'Ad-Astra a augmenté sa visibilité et a renforcé sa légitimité auprès d'acteurs décisionnels et exécutifs, l'adoption progressive d'une partie des critères d'évaluation qu'elle avait proposés, a généré des réactions d'hostilité à son égard. Les processus de réforme ont d'ailleurs continué à connaître des ralentissements peu compréhensibles, soumis aux aléas d'un fonctionnement politique opaque ${ }^{19}$.

Pour dépasser ces blocages et en prévision de l'adhésion de la Roumanie à I'Union européenne, $A d$-Astra a organisé une pétition qui défend l'adoption de réformes réelles et l'instauration d'un système d'évaluation et de promotion des chercheurs selon les pratiques internationales. Signée (en ligne) par 466 scientifiques, cette pétition a été envoyée en décembre 2006 à l'Autorité nationale pour la recherche scientifique (ANCS), au ministère de l'Éducation et de la recherche (MEdC) et au président de la Roumanie. En 2007, une commission présidentielle pour l'analyse et l'élaboration des politiques dans le domaine de l'éducation et de la recherche a été constituée. Présidée par l'ancien ministre de l'Éducation et de la recherche favorable à la réforme, cette commission compte aussi trois membres de l'association. Un des membres très

\footnotetext{
${ }^{19}$ II est difficile d'apprécier la nature et les principes sur lesquels reposent ces engrenages sans tomber dans le piège d'un jugement abusif. Les incidents relatés par certains membres d'Ad-Astra sur la liste de discussion laissent toutefois croire que les changements réclamés par l'association ont provoqué parfois des réactions démesurées (menaces, campagnes de diffamation...) de la part de certains acteurs qui avaient auparavant une grande influence sur les processus de distribution des subsides de recherche. En outre, l'instabilité politique a également contribué à ralentir la réforme.
} 
actifs du réseau $A d$-Astra, âgé de 35 ans, directeur de recherches dans une université allemande, a été nommé vice-président, tandis que le directeur exécutif de l'association est devenu le secrétaire de la commission. L'inclusion de ces membres Ad-Astra dans une structure chargée de collaborer avec le ministère de l'Éducation et de la recherche pour l'élaboration d'une stratégie efficace de réforme a permis d'intégrer une partie des idées, des valeurs et des principes promus par le groupe dans les documents, les analyses et les stratégies produites et proposées par cette commission.

\section{L'e-diaspora scientifique : une alternative concrète pour la valorisation des ressources des élites migrantes}

L'exemple d'Ad-Astra montre comment un réseau virtuel déterritorialisé peut enclencher des débats qui se propagent dans la société civile roumaine, engageant des changements de fond du système de la recherche. De fait, le réseau virtuel constitue un vecteur clé d'échanges scientifiques, sociaux, culturels et politiques, entre ses membres d'une part, et entre le réseau et d'autres structures sociales et politiques de la société d'origine d'autre part. Les chercheurs expatriés faisant partie d'Ad-Astra participent à la diffusion de valeurs démocratiques directement déduites de leurs expériences internationales, voire transnationales. Par ailleurs, si l'action transnationale de ce réseau est orientée vers l'espace national, ses buts visent, au contraire, une dé-essentialisation de la conception nationale de la recherche, par l'adoption des critères d'excellence reconnus au sein de la communauté internationale. En même temps, les pratiques activistes du réseau créent une unité extraterritoriale et stimulent le développement d'un sens civique. Mais si internet facilite essentiellement la propagation de valeurs et d'activités transnationales dans un champ englobant qui associe migrants et non-migrants, les transferts et la capitalisation de connaissances, d'expertises et d'expériences ne se réalisent que par la symbiose des formes d'action online et offline. Dans ce contexte, le rôle des membres du réseau qui se trouvent en Roumanie devient essentiel pour la mise en pratique des idées émergées dans l'espace virtuel. Plus concrètement, la contribution des scientifiques roumains expatriés à l'amélioration du système de la recherche roumain est consolidée par la collaboration qui s'établit entre eux et leurs confrères restés au pays. L'écho que les initiatives d'Ad-Astra ont trouvé plus largement dans les milieux de la recherche du pays et auprès d'autres acteurs de la société civile, a permis de transformer le capital culturel et social, d'expertise et de savoir-faire de ces migrants en une source directement exploitable pour la mise sur pied de projets. 
Néanmoins, indissociablement liée à l'efficacité de ces actions, la réceptivité des milieux politiques et institutionnels reste fondamentale pour la capitalisation de ressources incorporées par les chercheurs roumains de l'étranger. En fait, pour que les projets initiés par les uns et les autres puissent conduire à des actions concertées, il est capital qu'ils répondent tant aux intérêts de l'État qu'à ceux des chercheurs du pays, ainsi qu'aux aspirations des scientifiques expatriés.

Cette étude a révélé également l'émergence de nouvelles formes d'interaction et d'appartenance dans un monde d'interconnexions et d'interdépendances, dans lequel les frontières entre mobiles et sédentaires, migrants et non-migrants, dedans et dehors s'estompent progressivement. Plus particulièrement, elle a mis en évidence une transformation radicale de la façon d'être ensemble pour les migrants et les non-migrants et de leur capacité d'interagir, de s'organiser transnationalement et d'agir localement à distance. La recherche a révélé aussi qu'à l'ère du numérique, les nouvelles générations de migrants développent une culture du lien nouvelle, étant capables d'être « ici et là-bas » simultanément.

Cette réalité produit des dynamiques transnationales inédites, qui représentent un défi majeur, tant pour l'État-nation que pour les sciences sociales. Le migrant online devient une figure centrale de la modernité, acteur privilégié des processus de cosmopolitisation d'un quotidien dans lequel les échelles territoriales, politiques et sociales sont fortement enchevêtrées (Nedelcu, 2009). Le numérique est en train de produire une mutation profonde des structures sociales et du sens de l'appartenance, de la mobilité et des mouvements migratoires. Repenser la relation entre les sphères nationale et transnationale semble dès lors urgent, afin de développer plutôt une optique cosmopolitique inclusive both here and there, qu'une vision exclusive de type neither...or (Beck, 2006).

\section{Références bibliographiques}

BARRÉ R., V. HERNANDEZ, J.-B. MEYER et D. VINCK, 2003, Diasporas scientifiques - Scientific diasporas, coll. Expertise collégiale, Paris, IRD Éditions.

BECK U., 2006, Qu'est-ce que le cosmopolitisme ?, Paris, Aubier.

CLIFFORD J., 1997, Routes: Travel and Translation in the Late Twentieth Century, Cambridge, Harvard University Press. 
DE HAAS H., 2006, Engaging Diasporas. How governments and development agencies can support diaspora involvement in the development of origin countries, International Migration Institute, Oxford, University of Oxford.

FINDLAY A., 1988, "From settlers to skilled transients: the changing structure of British international migration », Geoforum 19 (4), pp.401-410.

FLORIAN R., 2004, "Migratia cercetatorilor romani. Situatia actuala, cauze, $\begin{array}{lllll}\text { solutii », } \quad A d \quad \text { Astra } 3 & \text { (2), 2004. http://www.ad- }\end{array}$ astra.ro/journal/6/florian migratia.pdf

FRANGOPOL P., 2002, Mediocritate si excelenta. O radiografie a stiintei si invatamantului din Romania, Bucuresti, Ed. Albatros.

GAILLARD A.-M. and J. GAILLARD, 2002, "Fuite des cerveaux, circulation des compétences et développement : un enjeu politique ", Mots pluriels, 20, février 2002. http://motspluriels.arts.uwa.edu.au/MP2002ajg.html.

GAILLARD J. et A.-M. GAILLARD, 1998, International migration of the highly qualified: A bibliographic and conceptual itinerary, New York, Center for Migration Studies.

HAIDUC I., 2002, "Cercetarea stiintifica in Romania oglindita intr-un recent raport American», Curierul de fizica no. 42, Bucuresti. http://www.fhh.org.ro/ed fhh/reviste/arhiva cdf/uncdf.htm

HÉAS S. et V. POUTRAIN, 2003, "Les méthodes d'enquête qualitative sur internet », ethnographiques.org, $\mathrm{n}^{\circ} 4$, http://www.ethnographiques.org/2003 /Heas,Poutrain.thml

LAZARSFELD P. et R. K. MERTON, 1954, «Friendship as a social process: A substantive and methodological analysis ", in M. BERGER, T. ABEL et C. PAGE (dir), Freedom and control in modern society, New York, Van Nostrand, pp.18-66.

LAZAROIU S. et al., 2004, Volume IV - Romania. More "Out » than "In " at the Crossroads between Europe and the Balkans, European Commission Project "Sharing experience: Migration trends in selected applicant countries and lessons learned from the "new countries of immigration" in the EU and Austria », Genève, OIM.

LOWELL B.L. et A. FINDLAY, 2002, Migration of highly skilled persons from developing countries: Impact and policy responses. International Migration Papers 44. Genève, International Labour Office, International Migration Branch.

http://www.ilo.org/public/English/protection/migrant/download/imp/imp44.pdf 
MEYER J.-B. et J. CHARUM, 1995, "La "fuite des cerveaux" est-elle épuisée ? Paradigme perdu et nouvelles perspectives ", Cahiers des sciences humaines, vol. 31, $\mathrm{n}^{\circ} 4$, pp.1003-1017.

MEYER J.-B. et V. HERNANDEZ, 2004, "Les diasporas scientifiques et techniques: état des lieux », dans M. NEDELCU (dir), La mobilité internationale des compétences. Situations récentes, approches nouvelles, Paris, L'Harmattan, coll. «Questions Sociologiques », pp.19-58.

MEYER J.-B., 2007, Building sustainability: The new frontier of diaspora knowledge networks, COMCAD, Working Paper 35, Bielefeld.

NEDELCU M., 2004, « Vers une nouvelle culture du lien : les e-pratiques locales et transnationales des migrants roumains hautement qualifiés», dans M. NEDELCU (dir), La mobilité internationale des compétences. Situations récentes, approches nouvelles, Paris, L'Harmattan, coll. "Questions Sociologiques », pp.9-17.

NEDELCU M., 2008, Néo-cosmopolitismes, modèles migratoires et actions transnationales à l'ère du numérique. Les migrants roumains hautement qualifiés, Thèse de doctorat, Université de Neuchâtel.

NEDELCU M., 2009, Le migrant online. Nouveaux modèles migratoires à l'ère du numérique, Paris, L'Harmattan.

POTOT S., 2003, Circulation et réseaux de migrants roumains : une contribution à l'étude des nouvelles mobilités en Europe. Thèse de doctorat. Université de Nice. http://tel.ccsd.cnrs.fr/documents/archives0/00/00/34/80/index fr.html

SALT J. et A. FINDLAY, 1989, «Les migrations internationales de personnel de haut niveau. Analyse théorique et incidences sur le développement », dans $\mathrm{R}$. Appleyard (dir) L'incidence des migrations internationales sur les pays en développement, Paris, OCDE, pp.181-218.

SALT J. J. CLARKE et P. WANNER, 2005, Les migrations internationales de la main d'œuvre, Études démographiques $n^{\circ} 44$, Strasbourg, Éditions du Conseil de l'Europe.

SAXENIAN A., 2000, Brain drain or Brain circulation ? The Silicon Valley-Asia connection. Harvard University, Asia Center : Modern Asia Series.

WICKRAMASEKARA P., 2004, "Les options politiques en réponse à la migration des compétences: rétention, retour et circulation» dans $M$. NEDELCU (dir), La mobilité internationale des compétences. Situations récentes, approches nouvelles, Paris, L'Harmattan, coll. "Questions Sociologiques », pp.167-201. 